\title{
Modélisation prospective et spécificités de la politique énergétique française
}

\author{
Nadia Maïzi et Edi Assoumou \\ Centre de Mathématiques Appliquées - Ecole des Mines de Paris - BP 207 - 06904 Sophia-Antipolis cedex -- nadia.maizi@ensmp.fr \\ Cet article, avant relecture et correction pour la revue J3EA, a fait l'objet d'une communication aux journées 2007 de la section \\ électrotechnique sur le thème Energie et Développement Durable à l'antenne de Bretagne de l'ENS de Cachan les 14 et 15 mars 2007.
}

\begin{abstract}
Résumé-Les stratèges qui se préoccupent des questions liées à l'Energie, doivent, dans un contexte international complexe, se doter d'outils normatifs performants pour faire face à des contraintes multiformes (quotas d'émissions, taxes, ouverture des marchés, raréfaction de la ressource). Dans cet article, nous montrerons comment une optimisation technico-économique de la chaîne énergétique, au moyen du modèle MARKALFrance, permet à l'horizon 2050, une approche prospective pertinente des conséquences environnementales de différentes options de la politique énergétique française. En particulier, l'impact environnemental de ces choix sera évalué à travers le niveau des émissions de $\mathrm{CO}_{2}$.
\end{abstract}

Mots clefs - prospective, planification, énergie, environnement, modèles d'optimisation bottom-up.

Connaissances requises - programmation linéaire, optimisation, énergie : notions d e base.

Niveau requis - second cycle universitaire, école d'ingénieur

\section{INTRODUCTION}

La déréglementation des marchés bouleverse le monde de l'énergie. Les acteurs traditionnels doivent désormais s'adapter à la nouvelle donne concurrentielle dans un contexte mondial où les ressources peu coûteuses et les surcapacités sont vouées à disparaître. Par ailleurs, la pression environnementale s'accroît. La suppression des émissions de soufre, l'exigence d'un ratio minimum d'énergies renouvelables en Europe, la restriction des émissions de gaz à effet de serre ... contraignent l'offre des acteurs au travers de mécanismes nombreux, complexes et non encore définitifs.

Face à cette complexité, les conséquences et impacts des choix stratégiques sont difficilement appréciables «par dires d'experts » et l'exercice de prospective recourant à la modélisation, l'optimisation et l'aide à la décision, devient un outil normatif précieux. Car tant pour les stratégies d'entreprises que pour les décisions politiques, cet exercice permet d'évaluer pour reprendre la formule de Pierre Massé, « un souhaitable qui apparaisse plausible à l'esprit prospectif et qui devienne probable pour l'esprit attaché à sa réalisation $\gg$.

Comme en témoigne le recensement fourni par un rapport du Plan [1], il existe de nombreuses approches utilisées à des fins de prospective énergétique, réparties suivant trois grandes classes : les modèles IAM (Integrated Assessment Model) [2], [3], les modèles économiques top-down (pour approche descendante) [4] et les modèles technologiques bottom-up (pour approche ascendante) [5].

Cependant, pour analyser les principaux déterminants du système énergétique à une échelle régionale donnée, pour anticiper les évolutions et les impacts des prix des énergies à moyen et long terme, pour estimer les émissions de polluants, toutes ces approches ne sont pas équivalentes. Car sans un modèle de la chaîne énergétique suffisamment agrégé pour intégrer les stratégies proposées et suffisamment détaillé pour prendre en compte les contraintes techniques et environnementales, on ne saurait répondre. Les modèles bottom-up de type MARKAL ${ }^{1}$ [6] (MARKAL pour Market Allocation) - outils ouverts et utilisés par une large communauté internationale $[7]^{2}$ - offrent, pour partie, une réponse à ce besoin.

Dans ce qui suit, nous proposons d'illustrer comment le choix de MARKAL à des fins prospectives permet d'éclairer les conséquences environnementales de choix politiques.

Tout d'abord, nous décrirons les principes sur lesquels repose cette modélisation en insistant sur les avantages qu'offre une approche technologique. Nous rappellerons ensuite le contexte français en matière de politique énergétique et ses spécificités. Enfin, nous illustrerons l'apport du modèle pour le traitement de quelques questions relatives aux émissions de $\mathrm{CO}_{2}$ à l'horizon 2050.

\section{LE RECOURS A LA MODELISATION PROSPECTIVE}

\section{A. Le modèle MARKAL}

Les modèles MARKAL permettent l'optimisation d'un coût technique, économique ou environnemental associé à une représentation technico-économique du système énergétique. Cette optimisation peut être réalisée à moyen et long terme pour des échelles spatiales différentes (régionale,

\footnotetext{
1 Derrière 1'appellation MARKAL on comprendra MARKAL/TIMES dernière version du modèle.

${ }^{2}$ Ce modèle développé sous l'égide de l'AIE (Agence Internationale de l'Energie), est aujourd'hui utilisé par quelques 77 équipes de modélisation dans plus de 37 pays ce qui permet les échanges au sein d'une large communauté internationale d'utilisateurs.
} 
nationale, mondiale...).

Dans MARKAL, les secteurs que l'on veut analyser sont considérés comme des chaînes de transformations subies par des ressources primaires énergétiques dans le but de satisfaire différentes demandes finales de services. La chaîne énergétique est décrite de l'amont (production et offre énergétique) à l'aval (secteurs économiques utilisant l'énergie finale) en passant par tous les secteurs intermédiaires consommateurs ou producteurs d'énergie comme l'illustre la figure 1. Dans cette représentation, une technologie est définie comme un convertisseur de « vecteurs énergétiques » et est associée à chaque étape de transformation.

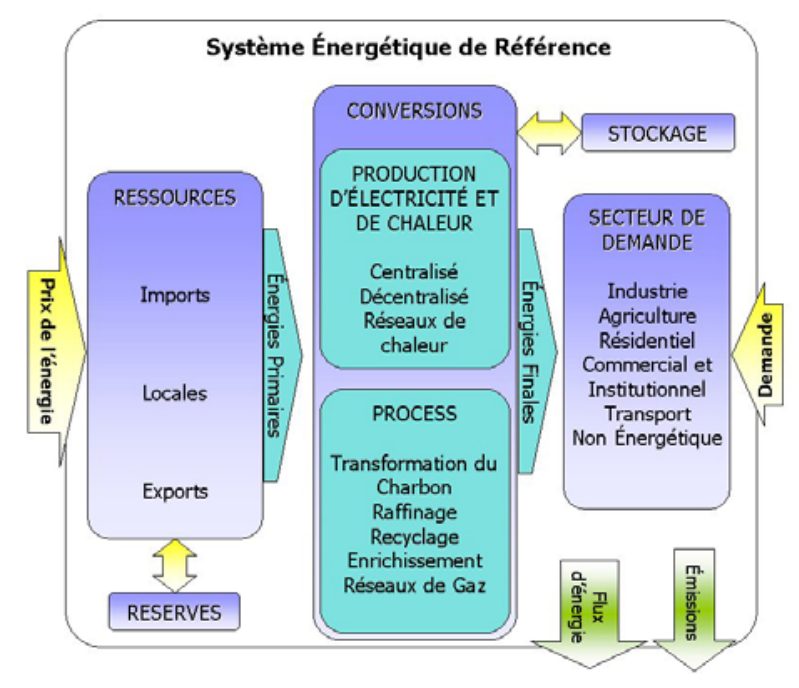

Fig. 1. Le système énergétique de référence

\section{B. Potentialités du modèle MARKAL}

La représentation technologique désagrégée décrite cidessus, constitue l'atout principal de l'approche MARKAL.

En premier lieu, cette approche permet de créer une économie virtuelle où les différentes technologies sont mises en concurrence. Concrètement, une formulation explicite des relations inputs outputs pour chaque technologie, permet sur l'horizon choisi (30 à 50 ans) et pour une demande finale donnée, de minimiser le coût global actualisé et d'obtenir les niveaux d'activités et d'investissements correspondants.

En second lieu, elle permet d'évaluer les émissions de certains polluants de la famille des gaz à effet de serre et, audelà, de faire 1' "Analyse du Cycle de Vie » des scénarios envisagés. C'est une potentialité importante notamment si l'on veut obtenir :

- une description complète des modes de consommation d'énergie (incluant distribution, qualité et fiabilité de la fourniture),

- une analyse précise des substitutions entre les formes et vecteurs d'énergie,

- une interprétation de la notion de besoin énergétique en termes de service et d'équipement,

- une meilleure évaluation des énergies renouvelables.

Enfin, il est possible à partir de données technicoéconomiques et d'hypothèses exogènes sur les tendances (demande, prix des ressources, taux d'actualisation, etc.) de fournir les évolutions - et leurs variantes - (typiquement tous les 5 ans jusqu'en 2050) des principaux déterminants du système énergétique, à savoir :

- l'impact des prix des énergies à moyen et long terme

- l'estimation des émissions de polluants

- la simulation des différentes compétitions technologiques et économiques

- la prise en compte de certaines mesures incitatives de réduction des émissions de gaz à effet de serre

- l'effet de ruptures technologiques

- le rôle des mesures liées à la maîtrise de la demande d'énergie

- l'impact du secteur des transports

- l'impact de différents projets de recherche et développement (performances énergétiques, projets d'énergies renouvelables, maîtrise de la demande d'électricité...)

- les conséquences des politiques énergétiques (par exemple les objectifs du protocole de Kyoto ont été envisagés via MARKAL pour la planification nationale de l'Inde, du Canada, de la Suisse, ... [8]).

Décliné au niveau français, nous allons voir les enjeux que le modèle MARKAL permet d'aborder.

\section{LES SPECIFICITES FRANÇAISES}

Comme le rappelle le livre blanc sur les énergies [9], la politique énergétique française de l'après guerre a connu trois grandes périodes :

"Les années 50 ont été marquées par le souci d'une énergie nationale et abondante : charbon et hydroélectricité, les années 60 par la recherche d'une énergie à meilleur marché : le pétrole. Les crises pétrolières des années 70 ont conduit à privilégier davantage l'indépendance stratégique et le rééquilibrage de notre balance commerciale ».

Il s'en est suivi le lancement, il y a 30 ans, d'un programme nucléaire de grande envergure (50 GW ont été mis en service entre 1980 et 1990 [10]) faisant du paysage énergétique français une exception au niveau mondial.

\section{A. Le secteur de la production d'électricité}

La figure 2. [11]-[12], illustre la répartition de la production électrique française pour l'année 2005 : 78,27\% est d'origine nucléaire, $10.23 \%$ est d'origine hydraulique et environ $10 \%$ d'origine thermique. Cette part de production thermique joue un rôle essentiel dans la gestion du réseau (pointe, besoins d'ajustement, services...).

Cette répartition est fondamentalement différente de la répartition mondiale de la production d'électricité [14] comme le montre la figure 3 . 


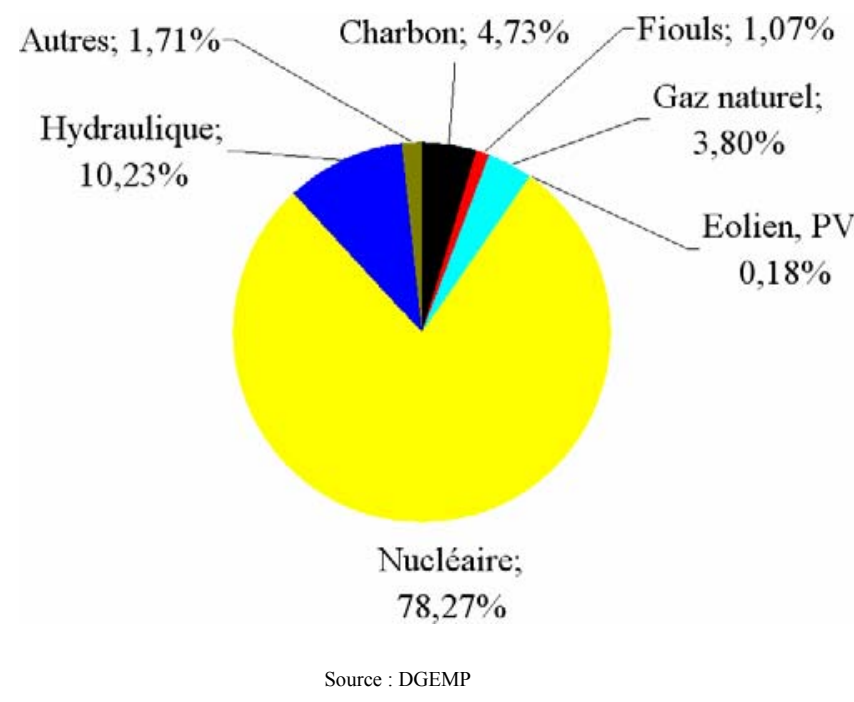

Fig. 2. Production électrique française 2005

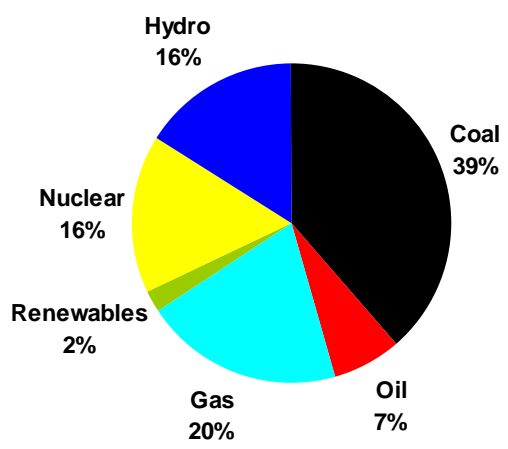

Fig. 3. Production électrique mondiale en 2004 (17,4 TWh)[14]

Par ailleurs, on peut noter que pour une durée de vie de 40 ans des installations existantes [18], le déclassement du parc électronucléaire français conduira dans les années 2020 à une baisse brutale de la capacité totale installée en 2000 . C'est l'effet falaise illustré par la figure 4.

EVOLUTION DE LA CAPACITE NUCLEAIRE EXISTANTE (40 ans durée de vie)

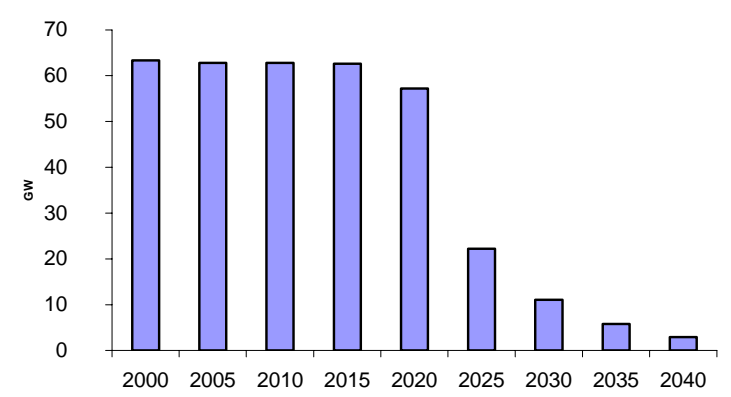

Fig. 4. Déclassement du parc électronucléaire

\section{B. Les émissions de GES (Gaz à Effet de Serre)}

Pour l'année 2000, la totalité des émissions en équivalent $\mathrm{CO}_{2}$, pour les usages liés à la consommation d'énergie de l'ensemble des secteurs de l'offre et de la demande, s'est chiffrée à $440 \mathrm{Mt}$ [13]. Comme pour la production d'électricité, la France se singularise par une contribution des secteurs les plus émissifs inversée par rapport à la moyenne mondiale représentée figure 5. [14]. En effet, le secteur des transports est le premier contributeur à ces émissions $(32,6 \%)$, suivi par l'industrie $(26,7 \%)$ et les secteurs résidentiel et tertiaire $(21,7 \%)$. La part de la production d'électricité et de chaleur ne compte que pour $10,1 \%$, ce qui souligne le caractère faiblement émissif de ce secteur, conséquence du choix d'une option nucléaire prépondérante pour la France.

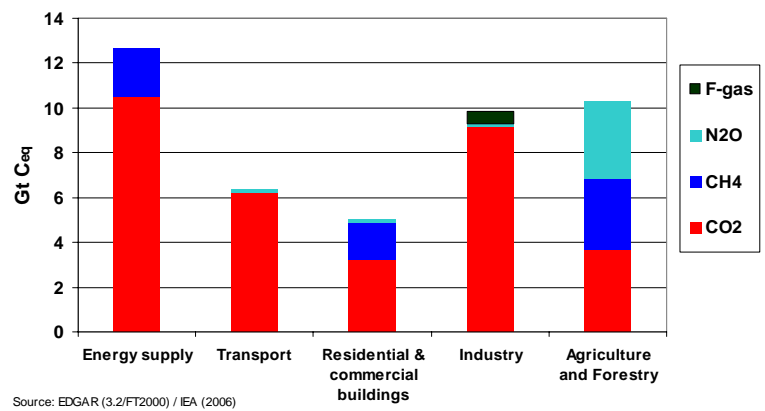

Fig. 5. Décomposition sectorielle des émissions mondiales de GES 2004

\section{Les questions de prospective}

Ces singularités françaises conditionnent les orientations de la stratégie énergétique de la France à l'horizon 2050 et en particulier de l'évolution du mix énergétique dans un contexte où les engagements nationaux au niveau environnemental sont importants :

- dans le cadre du protocole de Kyoto, stabiliser les émissions de GES en 2010 par rapport à leur niveau en 1990

- au niveau européen :

- assurer à hauteur de $21 \%$ la contribution des renouvelables dans la consommation domestique d'électricité en 2010

- diminuer de 20\% les émissions globales (sans déclinaison nationale) de GES en Europe à l'horizon 2020 par rapport à leur niveau de 1990

- au niveau national réduire par 4 les émissions de GES par habitant à l'horizon 2050 par rapport à leur niveau de 1990.

C'est notamment pour évaluer les conséquences de ces engagements internationaux que nous avons développé le modèle MARKAL-France.

\section{APPLICATIONS DU MODELE MARKAL-FRANCE}

Afin d'illustrer les possibilités de l'approche MARKAL nous la déclinerons pour l'ensemble des secteurs contributifs aux émissions. Puis, nous nous focaliserons sur l'étude spécifique de deux secteurs particuliers :

1. le secteur des transports, plus grand contributeur aux émissions, et qui présente donc un intérêt certain pour le déploiement d'une politique de maîtrise des émissions ;

2. le secteur électrique, singulier par son orientation 
nucléaire, et remarquable par le poids de la part thermique dans le bilan global de ses émissions.

\section{A. Le scénario de base pour la France}

Afin d'établir une référence pour la comparaison entre scénarios, nous avons bâti un scénario, dit scénario de base. Il repose sur les hypothèses suivantes :

1. absence de contraintes sur les émissions de GES

2. pas de contraintes environnementales

3. un taux d'actualisation de $8 \%$

4. une projection de la croissance de la demande pour tous les services avec globalement: une croissance rapide de la demande de mobilité dans les transports, une croissance soutenue pour l'industrie, une croissance plus lente pour le résidentiel avec une stagnation de la population et du nombre de logements

5. une évolution des prix des ressources fossiles données Tableau $1^{3}$

\begin{tabular}{|l|r|r|r|r|}
\cline { 2 - 5 } \multicolumn{1}{c|}{} & 2000 & 2005 & 2020 & 2050 \\
\hline pétrole (\$/baril) & 27,89 & 54 & 48 & 60 \\
\hline gaz (\$/MBTU) & 2,78 & 5,24 & 6,6 & 8 \\
\hline charbon (\$/tonnes) & 34,93 & 61,3 & 67 & 71 \\
\hline
\end{tabular}

TABLEAU 1. HYPOTHESE DE PRIX D'IMPORTATION DES RESSOURCES FOSSILES

6. des investissements dans les nouveaux réacteurs nucléaires au rythme : d'un prototype (1.5 GW) en 2012, d'une période d'observation entre 2012 et 2020, d'un maximum de nouvelles capacités installées de $3 \mathrm{GW} /$ an après 2020.

On peut remarquer que

- l'hypothèse 4 a pour conséquence la diminution de la demande en énergie utile pour le chauffage individuel, à cause de la rénovation du bâtiment ancien, et d'une meilleure efficacité dans le neuf;

- l'hypothèse 6 sur le développement du nucléaire prolonge le rythme d'intégration de nouvelles capacités de production tel qu'établi dans le rapport prévisionnel pour le remplacement des réacteurs existants [18] sur la période 2020-2030.

\section{B. Consommation globale d'énergie primaire et émissions de CO2 dans le scénario de base}

MARKAL-France permet d'évaluer l'évolution du mix énergétique fournissant l'ensemble des secteurs consommateurs d'énergie jusqu'en 2050. Cette évolution de la consommation totale d'énergie primaire est donnée figure 6.

\footnotetext{
${ }^{3}$ Ces hypothèses de prix sont celles retenues dans le cadre du projet européen NEEDS où est réalisée la modélisation de l'Europe des 25. La fluctuation du prix du pétrole fait l'hypothèse d'une évolution cyclique.
}

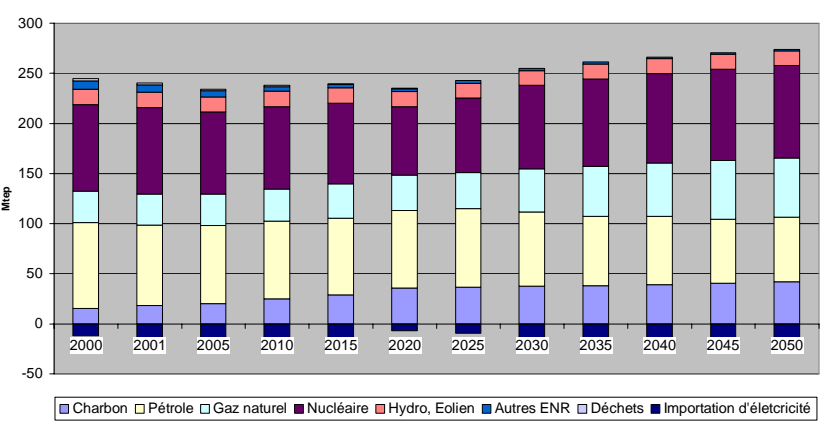

Fig. 6. Consommation en énergie primaire pour la France (usages énergétiques)

Les principaux résultats sont :

- une baisse à moyen terme (2020-2030) de la part du nucléaire avec le déclassement des centrales existantes, suivi dans le plus long terme d'une mise en service progressive de centrales EPR ;

- une compensation de cette baisse à moyen terme par une réduction des exportations ;

- une augmentation de la part du charbon avec l'évolution des prix du pétrole ;

- une augmentation de la part du gaz naturel.

Pour ces résultats, MARKAL évalue les émissions de $\mathrm{CO}_{2}$ associées aux différents secteurs émissifs sur l'horizon envisagé figure 7. Les spécificités du système énergétique français se trouvent ainsi prolongées dans le scénario de base avec une augmentation des émissions de $\mathrm{CO}_{2}$ dans les transports et une contribution toujours faible du secteur de la production d'énergie.

Emissions de $\mathrm{CO} 2$

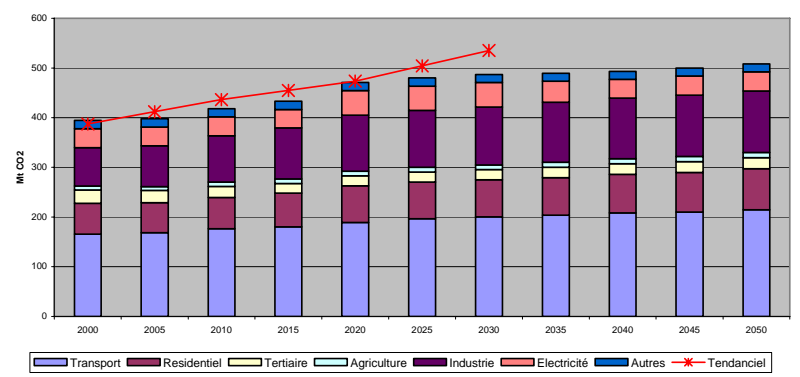

Fig. 7. Evolution des émissions de $\mathrm{CO} 2$

Nous avons positionné le scénario tendanciel DGEMP [18] représenté par la courbe rouge par rapport à ces résultats. A l'évidence, l'écart en tendance constaté impose de confronter dans le détail les hypothèses retenues pour chaque scénario. Mais il a l'avantage de mettre en exergue la différence de philosophies entre une «approche tendancielle » et une « approche de type MARKAL » :

- un tendanciel évalue les performances d'un système énergétique proche de celui de l'année de base et dans le prolongement des évolutions historiques. Son horizon de pertinence est plutôt la simulation de scénarios de faible rupture sur le court et le moyen terme (où les tendances historiques peuvent être réputées dominantes);

- $\quad$ une approche MARKAL-France optimise librement 
des choix technologiques en fonction de leurs performances coût/efficacité et en prenant en compte les meilleures technologies disponibles. L'intérêt économique de changements de technologies sur le moyen et le long terme est donc évalué.

Le scénario de base MARKAL n'est donc pas un tendanciel au sens du scénario DGEMP.

\section{Zoom sur le secteur des transports}

L'évolution du mix des transports dans le scénario de base est illustrée figure 8.

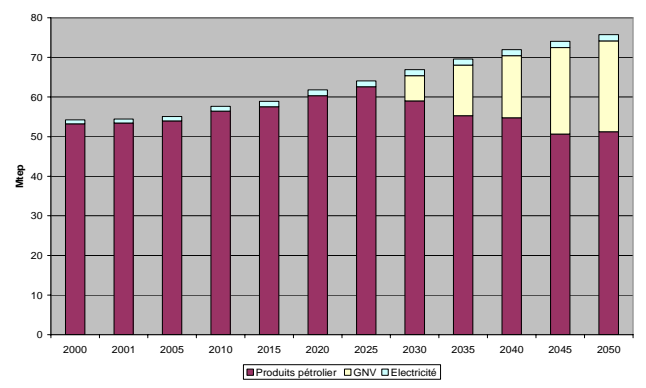

Fig. 8. Consommation d'énergie dans le secteur des transports

Dans ce scénario, la consommation d'énergie, après avoir stagné pendant les 15 premières années de l'horizon prospectif, croit de manière très significative après 2020. Cela traduit le fait que l'amélioration de l'efficacité énergétique est compensée par un accroissement de la demande de mobilité, notamment pour les transports maritimes et aériens pour lesquels l'usage du kérosène et du fioul lourd domine. Du fait du niveau des prix du pétrole, le gaz prend également une part significative pour les carburants utilisés pour le transport de marchandise à partir de 2030.

Malgré la substitution vertueuse (en termes d'émissions de $\mathrm{CO}_{2}$ ) du pétrole par du gaz naturel, ces résultats montrent que l'utilisation des technologies les plus compétitives économiquement, n'inverse pas réellement la tendance en termes d'émissions de $\mathrm{CO}_{2}$ du secteur des transports.

C'est pourquoi, il devient pertinent d'évaluer les technologies les plus rentables sous contraintes d'émissions de $\mathrm{CO}_{2}$. Il existe plusieurs possibilités de traduction de la contrainte $\mathrm{CO}_{2}$ dans MARKAL. Une première option consiste à imposer, pour un scénario alternatif, une contrainte maximale d'émissions et d'analyser sa pertinence et sa faisabilité en termes de chemin technologique.

La répartition de cette contrainte dans le temps influe fortement sur les investissements réalisés. Par exemple, l'étude [19] propose des chemins alternatifs, R1, R2, de la figure 9., pour atteindre l'objectif de réduction par 4 des émissions de CO2 (par rapport à leur niveau de 1990) à l'horizon 2050.

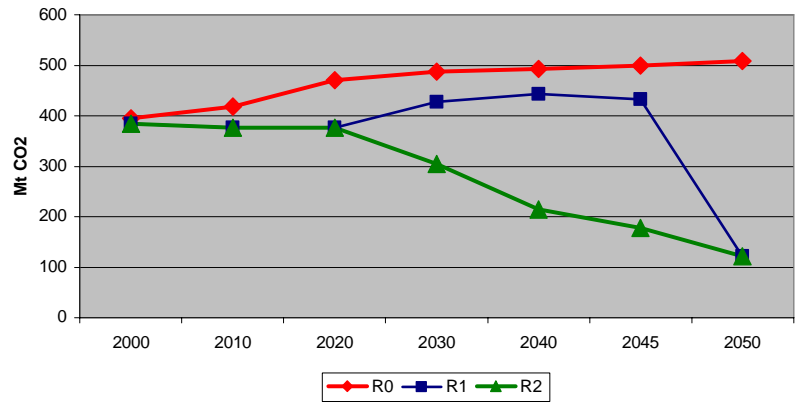

Fig. 9. Evolution des émissions de $\mathrm{CO}_{2}$ pour les consommations d'énergie

Le chemin R0 (rouge) correspond au scénario de base (« laisser faire ») tel qu'évalué figure 7. Le chemin R1 (bleu) a été obtenu en imposant une contrainte d'émission uniquement sur la dernière période de l'horizon. Enfin, le chemin R2 (vert) résulte d'un étalement dans le temps des contraintes d'émission.

A partir de ces profils d'émissions maximales, MARKALFrance calcule l'ajustement du mix énergétique de chacun des secteurs. Par exemple, la figure 10. représente l'évolution de la répartition par type (en tonne équivalent pétrole), des carburants dans le secteur des transports, dans l'hypothèse du chemin R2.

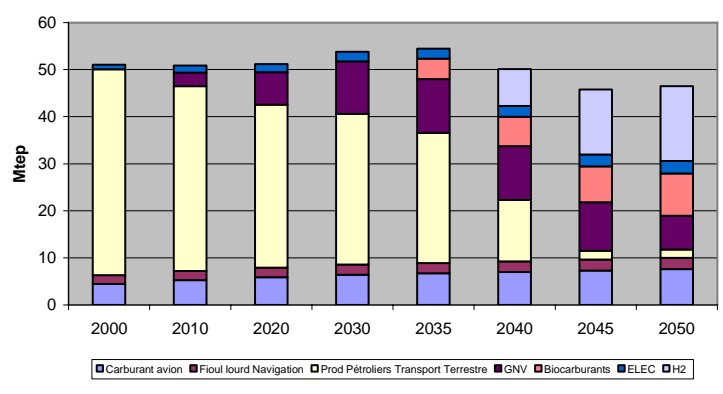

Fig. 10. Evolution de la répartition par type de carburant

On constate pour le scénario R2, l'objectif facteur 4 est atteint grâce à une transition vers l'hydrogène et les biocarburants, accompagnée d'une disparition quasi totale des carburants pétroliers pour le transport terrestre à partir de 2045.

\section{Raffinements pour le secteur de la production d'électricité}

La seconde spécificité de la France est le poids du parc électronucléaire dans la production électrique et son corollaire en termes de faibles émissions de $\mathrm{CO}_{2}$ du secteur électrique. Le scénario de base, représenté figure 6., maintient à l'horizon 2050 cette caractéristique avec un déploiement important de centrales de type EPR.

MARKAL offre la possibilité d'évaluer des évolutions alternatives. Les études [15]-[17] comparent deux variantes de politique de déploiement du nucléaire en France à l'horizon 2050 pour le secteur électrique : l'une sans limite de part de marché, l'autre avec une limitation à $50 \%$ de la part de marché du nucléaire à l'horizon 2050. Ces études portent cependant sur un modèle exclusivement électrique et reposent sur des hypothèses différentes de celles retenues 
pour le scénario de base ${ }^{4}$.

Les figures 13. et 14. montrent l'évolution du mix énergétique dans les deux cas. On constate que les technologies de substitution mises en place pour pallier la contrainte de déploiement sur le nucléaire s'appuient sur une ressource charbon (centrales à lit fluidisé), la plus rentable pour une production en base cf figure 15. Il est à noter que pour réaliser cette étude, nous avons utilisé une version améliorée de MARKAL, dite MARKAL-flexible [18], développée dans le but de pouvoir tenir compte de l'usage différencié de moyens de production d'électricité en fonction de la dynamique des appels.

Cette approche flexible permet d'analyser l'effet d'une contrainte $\mathrm{CO}_{2}$ sur un parc électrique français dont la part de moyens de production thermique est renforcée. Cette contrainte $\mathrm{CO}_{2}$ est traduite ici à travers une taxe de 20 $€ /$ tonne de $\mathrm{CO}_{2}$ émise. On constate que l'application de cette taxe modifie fortement la structure de la production thermique avec un arbitrage en faveur du gaz (figure 11). Les lits fluidisés sortent du parc de production et les cycles combinés assurent l'essentiel de la fourniture d'électricité. Ce scénario fait aussi apparaître dès 2015 une nouvelle technologie gaz avec des niveaux d'émissions faibles pour la production d'électricité en base : des centrales hybrides pile à combustible SOFC avec reformage du gaz /turbine à gaz [20].

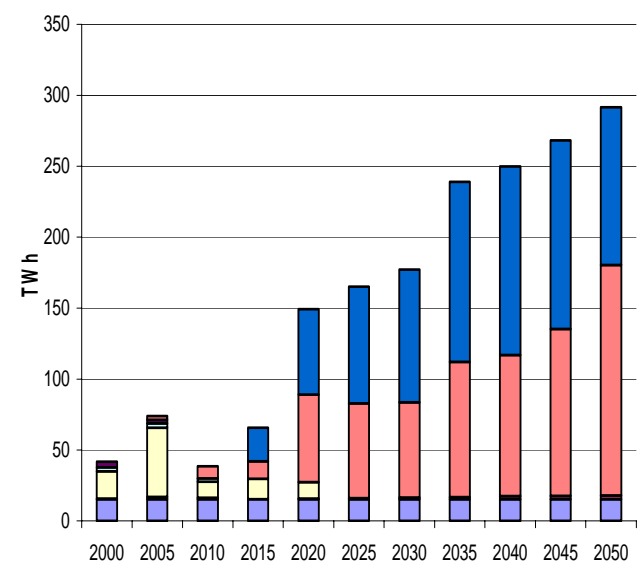

QDCN QTurb Gaz DCH_SC QCH_LF QTurb Fioul QCCG DPAC

DCN : Production décentralisée; Turb Gaz : Turbine à gaz ; $\mathrm{CH}$ SC : charbon super critique ; CH_LF : charbon lit fluidisé, Turb Fioul : Turbine fioul; CCG : cycle combiné à gaz; PAC : centrale hybride SOFC-turbine gaz

Fig. 11. Mix pour la production thermique: Effet d'une taxe $\mathrm{CO}_{2}$ de $20 € / \mathrm{t}$ sur le scénario EPR modéré

Ces technologies jouent un rôle important lorsque la

${ }^{4}$ Il s'agit principalement :

- de la demande d'électricité à 2030 qui est issue du volet électricité du scénario tendanciel 2004 de la DGEMP [21] et qui est ensuite prolongée à 2050 par le scénario $\mathrm{H} 1$ du rapport Charpin-Dessus-Pellat [22] ;

- des hypothèses de prix constants pour les ressources qui sont celles du scénario DGEMP 2004 (pétrole à 30\$/baril, gaz naturel à $4 \$ / \mathrm{Mbtu}$, charbon à $45 \$ / \mathrm{t})$.

Ces hypothèses, quoique simples permettent, dans un cadre normatif, d'isoler plus clairement (avant toute combinaison à d'autres effets) l'amplitude potentielle des changements induits. politique nucléaire est limitée : en effet, il faut répondre aux besoins de production en base et lorsque la taxe $\mathrm{CO}_{2}$ est prise en compte les cycles combinés sont pénalisés (les émissions sont de $353 \mathrm{gCO}_{2} / \mathrm{kWh}$ pour les cycles combinés contre $300 \mathrm{gCO}_{2} / \mathrm{kWh}$ pour les centrales hybrides). On voit donc émerger une nouvelle technologie qui devient compétitive.

Enfin, on peut observer l'évolution des émissions de $\mathrm{CO}_{2}$ sur tout l'horizon en fonction de la politique nucléaire retenue et de l'application ou non de la taxe environnementale. Les résultats représentés figure 12. dessinent quatre situations très contrastées conduisant en 2050 à des niveaux d'émission variant de 19,5 à 219,1 Mt de $\mathrm{CO}_{2}$.

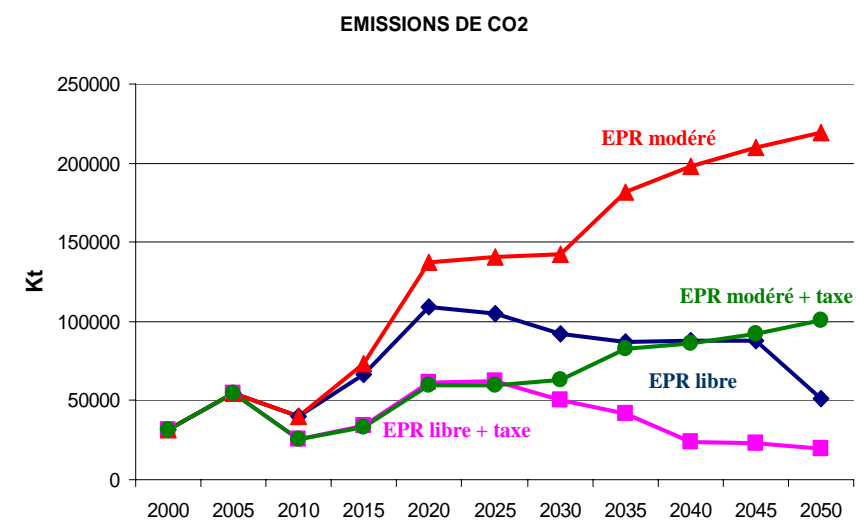

Fig. 12. Effet d'une taxe sur les émissions de $\mathrm{CO}_{2}$ du secteur de la production d'électricité

Avec 51,12 Mt de $\mathrm{CO}_{2}$, le scénario de déploiement de l'EPR conduit en 2050 à une augmentation de 60\% des émissions par rapport à $2000(31,66 \mathrm{Mt})$ avec un pic à 108,9 Mt en 2020. Lorsque la taxe environnementale est appliquée, les émissions baissent de $39 \%$ en 2050 (19,5 Mt) par rapport à 2000. Le pic d'émission de 2020-2025 n'est plus que de 61,3 Mt.

Un déploiement modéré de l'EPR conduit à un appel plus important des centrales thermiques. Les émissions croissent alors continuellement sur toute la période pour atteindre $219,1 \mathrm{Mt}$ en 2050 soit une multiplication par 6,9 du niveau de 2000. La taxe réduit fortement l'appel au charbon et donc les quantités de $\mathrm{CO}_{2}$ émises. Les émissions sont néanmoins multipliées par 3,2 par rapport à 2000 .

\section{CONCLUSION}

Grâce à la possibilité de définir des contraintes additionnelles de manière souple ou d'imposer des valeurs alternatives aux paramètres descriptifs du système énergétique, voire d'améliorer la représentation de certains secteurs spécifiques, MARKAL permet la simulation d'un large éventail de scénarios et l'analyse des choix technologiques correspondants. A travers quelques exemples nous avons illustré ces possibilités, notamment en combinant deux scénarios technologiques de déploiement futur des centrales nucléaires de type EPR à l'application ou non d'une taxe sur les émissions de $\mathrm{CO}_{2}$.

L'approche MARKAL qui sous tend cette étude a permis de proposer différentes alternatives de l'avenir énergétique; son usage peut être dérivé afin de permettre «l'initiation» 
de l'industrie française, pour qu'elle

- prépare ses futurs marchés en intégrant les enjeux environnementaux dans ses choix stratégiques

- intègre en amont des objectifs d'éco-conception.

Il faudra pour cela améliorer le réalisme de l'exercice prospectif et notamment pouvoir intégrer les modes de transport et de distribution de l'énergie, éléments d'arbitrage décisifs sur lesquels peuvent s'opérer des transferts de pollution. En ceci on dépasserait les méthodologies prospectives classiques, où les capacités de transport sont ajustées a posteriori aux scénarios d'offre et de demande.

\section{REMERCIEMENTS}

Nous remercions Marc Bordier (CMA/Mines Paris), Gilles Guerassimoff (CMA/Mines Paris) membres du projet MARKAL ainsi que Vincent Mazauric (Schneider Electric) pour les nombreuses discussions enrichissantes que nous avons partagées autour de ces sujets.

\section{REFERENCES}

[1] P.N. Giraud, N. Jestin-Fleury, "Effet de serre : modélisation économique et décision publique", Commissariat Général du Plan, France, 2002.

[2] J. Rotmans Jan, B. A. van Asselt Marjolein "Uncertainty Management in Integrated Assessment Modeling: Towards a Pluralistic Approach", Kluwer Academic Publishers, Environmental Monitoring and Assessment, 69 (2), p.101-130, June 2001.

[3] E. A. Parson and K. Fisher-Vanden, "Integrated assesment models of global climate change", Annual revue of Energy and Enviroment, $p$. 589-62, 1997.

[4] J. Percebois, "L'apport de la théorie économique aux débats énergétiques", Rapport CREDEN / Faculté de Sciences Economiques de l'Université de Montpellier I, 1999.

[5] J. Girod et J. Percebois, "Energie : fonctions de production et modèles de demande", dans Energie : modélisation et économétrie, Economica Ed. (1985), pp 423-443.

[6] Fishbone, L.G., H. Abilock, "MARKAL, a linear programming model for energy systems analysis: technical description of the BNL version", International journal of Energy research, vol. 5, pp 353-375, 1981.
[7] ETSAP (Energy Technology Systems Analysis Programme) Available: http://www.etsap.org

[8] R. Loulou, A. Kanudia, and D. Lavigne "GHG Abatement in Central Canada with Inter-provincial Cooperation", Energy Studies Review, Vol. 8, No. 2, pp. 120-129, 1996.

[9] "Livre blanc sur les énergies", Débat national sur les énergies, présenté par Nicole Fontaine (2003).

[10] C. Bataille et C. Biraux "La durée de vie des centrales nucléaires et les nouveaux types de réacteurs", Rapport No 290 du Sénat, 2003.

[11] Direction Générale de l'Energie et des Matières Premières, "Production-Distribution de l'énergie électrique en France et dans les régions en 2003 et 2004", 2002.

[12] Gestionnaire du réseau de transport d'électricité, "Résultats techniques du secteur électrique en France 2000", RTE, 2000.

[13] Centre Interprofessionnel Technique d'Etude de la Pollution Atmosphérique, "Inventaire des émissions de polluants atmosphériques en France", CITEPA 2006.

[14] H.-Holger Rogner "Nuclear Power And Climate Change", CoP-12, Nairobi, Kenya, November 2006.

[15] E. Assoumou, "Modélisation MARKAL pour la planification énergétique long terme dans le contexte français", Thèse de Doctorat de l'Ecole des Mines de Paris, 2006.

[16] N. Maïzi, E. Assoumou, M. Bordier, G. Guerassimoff, V. Mazauric "Key features of the electricity production sector through long-term planning: the French case", Power Systems Conference and Exposition, Atlanta 29 Oct - 01 Nov 2006.

[17] N. Maïzi, E. Assoumou, M. Bordier, G. Guerassimoff, V. Mazauric "Energy mix planning for the French electricity production sector", European Conference on Operational Research Euro2006, Reykjavik 2-5 July 2006.

[18] Direction Générale de l'Energie et des Matières Premières, "Scénario énergétique tendanciel à 2030 pour la France", DGEMP-OE 2004.

[19] E. Assoumou, M. Bordier, G. Guerassimoff, N. Maïzi, "Reducing greenhouse gas emissions by a factor of 4 by 2050: a bottom-up analysis of post Kyoto emissions targets for France", International Energy Workshop, Cape-Town 27-29 June 2006.

[20] Direction générale de l'énergie et des matières premières, "Coûts de référence de la production électrique, moyens de production décentralisés", DGEMP-DIDEME, 2004.

[21] Direction Générale de l'Energie et des Matières Premières, "Scénario énergétique tendanciel à 2030 pour la France", DGEMP-OE 2004.

[22] Charpin, J.M., Dessus, B., Pellat, R., "Etude économique prospective de la filière électrique nucléaire", Collection des rapports officiels, Premier ministre, 2000.

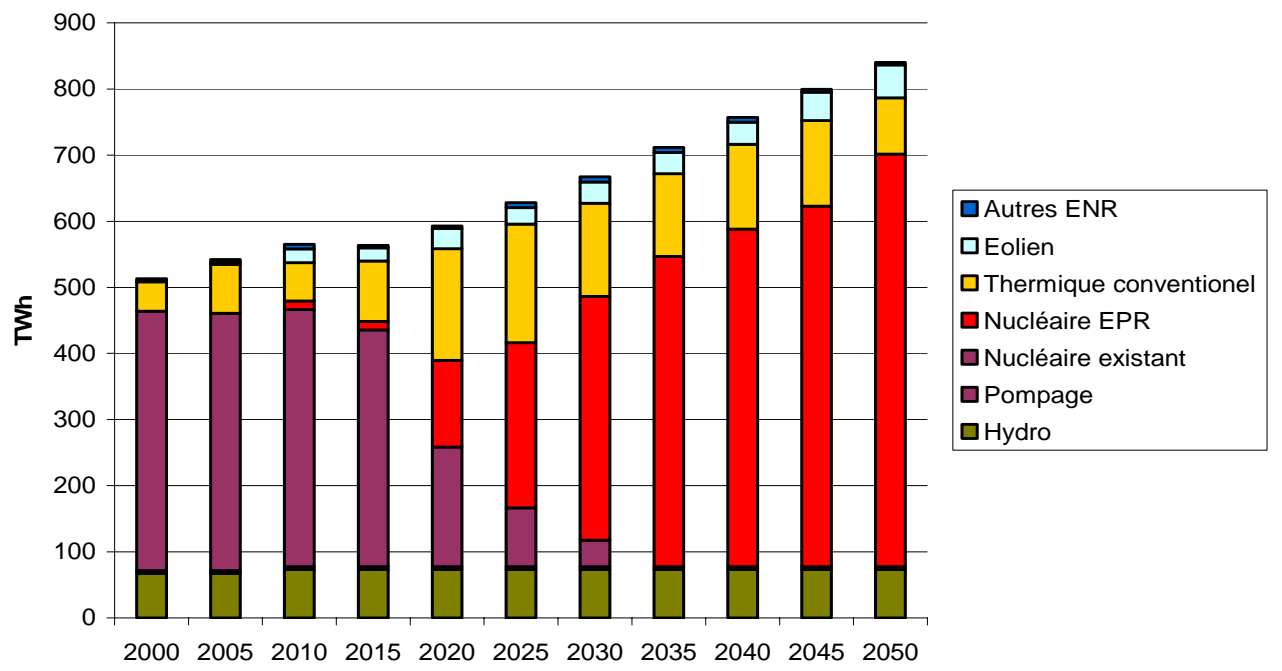

Fig. 13. Mix pour la production d'électricité : scénario de base 


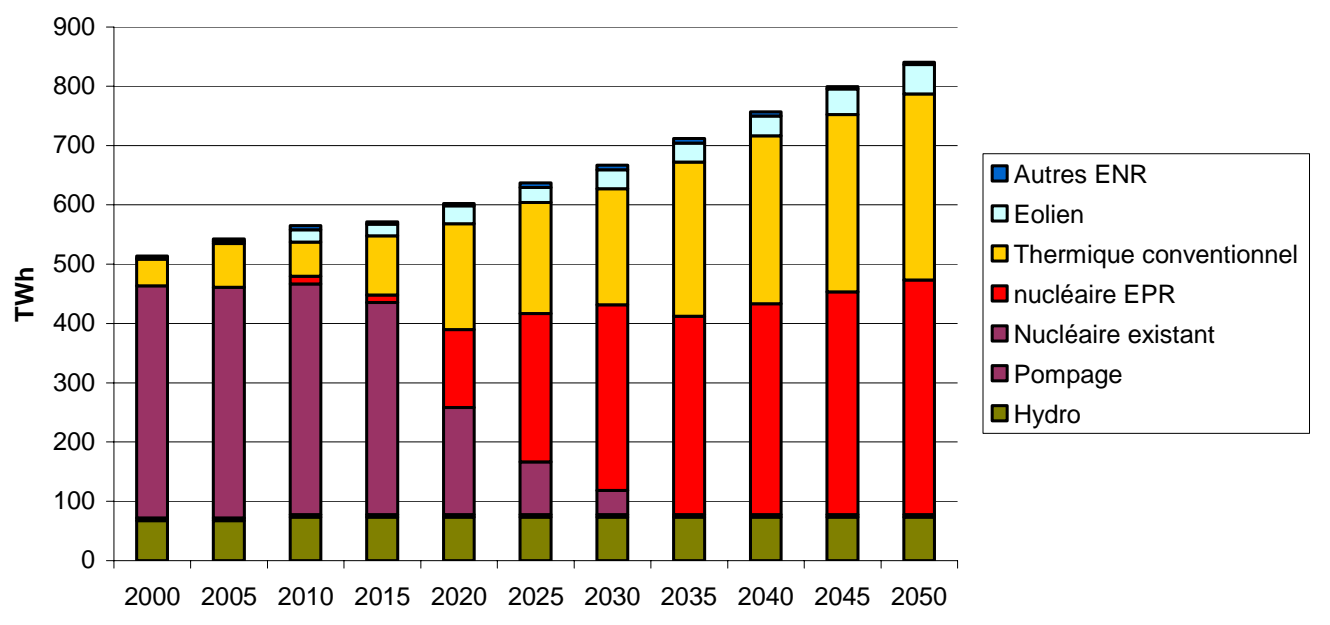

Fig. 14. Mix pour la production d'électricité : politique nucléaire modérée

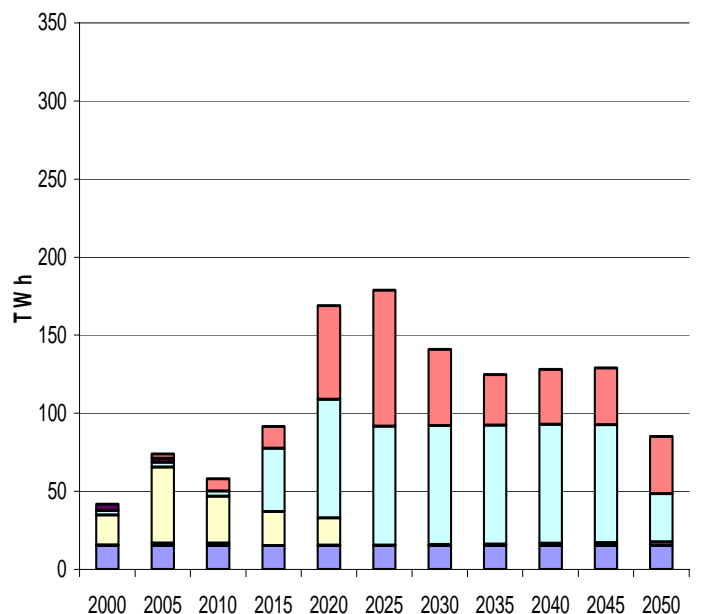

QDCN QTurb Gaz QCH_SC QCH_LF DTurb Fioul QCCG

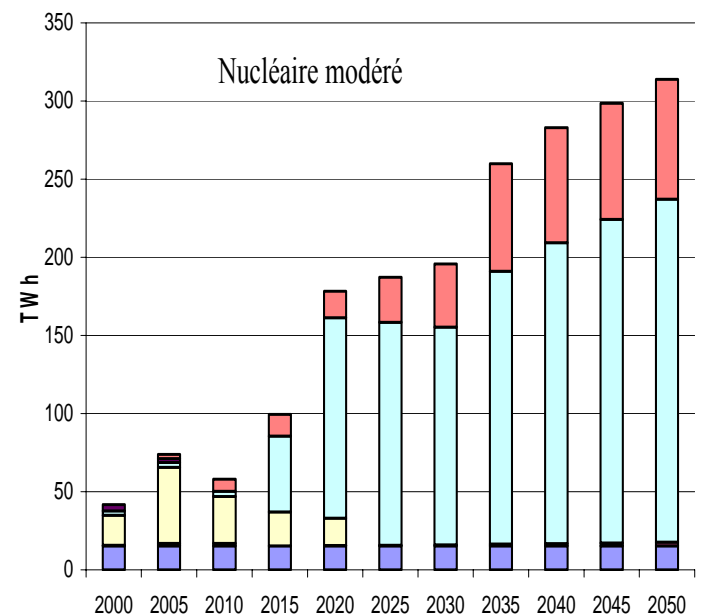

QDCN QTurb Gaz QCH SC QCH_LF QTurb Fioul QCCG

Fig. 15. Mix pour la production thermique avec ou sans contrainte EPR

DCN : Production décentralisée ; Turb Gaz : Turbine à gaz ; CH_SC : charbon super critique ; CH_LF : charbon lit fluidisé, Turb Fioul : Turbine fioul ; $\mathrm{CCG}$ : cycle combiné à gaz 


\section{BIBLIOGRAPHIE DES AUTEURS}

Nadia Maïzi, est Ingénieur civil de l'Ecole des Mines de Paris (P85), titulaire d'une Maitrise de Mathématiques Pures (Universié de Provence 1986), d'un DEA en Autmatique et Traitement du Signal option Image et Sciences de l'Univers de l'Université de Nice (1989) et d'un Doctorat de l'Ecole des Mines de Paris spécialité Informatique Temps Réel, Robotique et Automatique (1992). En 1994 elle a effectué un séjour post doctoral à l'Université de Stanford (Robotics Laboratory, Computer Science Department).

Elle est aujourd'hui Professeur de Mathématiques Appliquées à l'Ecole des Mines de Paris où elle dirige le Centre de Mathématiques Appliquées depuis Mai 2000.

Elle y a initié et anime des activités de recherche et d'enseignement liées à l'optimisation et l'aide à la décision dans le domaine de l'Economie, l'Energie et l'Environnement. Dans ce cadre, elle développe avec son équipe un modèle Markal de planification énergétique pour la France et travaille pour le Conseil d'Analyse Stratégique (Cabinet du Premier Ministre) à l'établissement d'un scénario «facteur 4 » à l'horizon 2050, pour l'Agence Internationale de l'Energie et est déléguée adjointe pour la France dans le cadre de l'Energy Technology Systems Analysis Programme (ETSAP).

Elle a également fondé le mastère spécialisé de l'Ecole des Mines de Paris en Ingénierie et Gestion de l'Energie OSE dont elle est responsable pédagogique pour le module Optimisation. Elle y enseigne la recherche opérationnelle, et intervient en tant qu'expert en optimisation auprès des partenaires industriels de la formation.

Elle a été adjointe à la direction de l'Institut Supérieur en Automatique et Informatique de l'Ecole des Mines de Paris de 1996 à 2000.

Elle est Membre de la SMAI (Société de Mathématiques Appliquées et Industrielles) et de la SIAM society.

Edi Assoumou est ingénieur ENSEEG-ENSIEG (2001) en production décentralisée et stockage de l'énergie et titulaire d'un mastère spécialisé en optimisation des systèmes énergétiques (2002) de l'Ecole des Mines de Paris (EMP). Il a soutenu en Juin 2006 une thèse à l'EMP sur le développement d'une modélisation MARKAL pour la prospective énergétique en France et notamment la représentation des systèmes électriques. Il a depuis rejoint le Centre de mathématiques Appliquées de l'Ecole des Mines de Paris où il travaille dans le projet de recherche et d'enseignement liées à l'optimisation et l'aide à la décision dans le domaine de l'Economie, l'Energie et l'Environnement. Il y est responsable de plusieurs projets de développement de modélisation prospective, notamment à l'échelle européenne (NEEDS (New Energy and Externalities Development for Sustainability)) et mondiale (Energy Technology Programme 2008). 